\title{
Control and Elimination of Cracking of AlGaN Using Low-Temperature AlGaN
}

\section{Interlayers}

J. Han, ${ }^{*}$ K. E. Waldrip, S. R. Lee, J. J. Figiel, S. J. Hearne, G. A. Petersen, S. M. Myers Sandia National Laboratories, Albuquerque, New Mexico 87185

\begin{abstract}
We demonstrate that the insertion of low-temperature (LT) AlGaN interlayers is effective in reducing mismatch-induced tensile stress and suppressing the formation of cracks during growth of AlGaN directly upon GaN epilayers. Stress evolution and relaxation is monitored using an in-situ optical stress sensor. The combination of in-situ and ex-situ characterization techniques enables us to determine the degree of pseudomorphism in the interlayers. It is observed that the elastic tensile mismatch between $\mathrm{AlGaN}$ and $\mathrm{GaN}$ is mediated by the relaxation of interlayers; the use of interlayers offers tunability in the in-plane lattice parameters.
\end{abstract}

\footnotetext{
*Electronic mail: jhan@sandia.gov
} 


\section{DISCLAIMER}

This report was prepared as an account of work sponsored by an agency of the United States Government. Neither the United States Government nor any agency thereof, nor any of their employees, make any warranty, express or implied, or assumes any legal liability or responsibility for the accuracy, completeness, or usefulness of any information, apparatus, product, or process disclosed, or represents that its use would not infringe privately owned rights. Reference herein to any specific commercial product, process, or service by trade name, trademark, manufacturer, or otherwise does not necessarily constitute or imply its endorsement, recommendation, or favoring by the United States Government or any agency thereof. The views and opinions of authors expressed herein do not necessarily state or reflect those of the United States Government or any agency thereof. 


\section{DISCLAIMER}

Portions of this document may be illegible in electronic image products. Images are produced from the best available original document. 
Most of the III-nitride devices to date have been grown on thick $(2 \sim 4 \mu \mathrm{m}) \mathrm{GaN}$ layers nucleated on heavily mismatched substrates (such as $\mathrm{Al}_{2} \mathrm{O}_{3}$ or $\mathrm{SiC}$ ). The thick GaN layer leads to improved structural quality and serves as a (nearly relaxed) pseudosubstrate on which active layers consisting of $\mathrm{AlGaInN}$ heterostructures are grown. Unlike zincblende semiconductors, misfit dislocations do not easily afford stress relief during strained heteroepitaxial growth of the (0001)-oriented, wurtzite, III-nitrides. Difficulty of misfit-dislocation glide in wurtzite crystals in response to a biaxial strain field is due to the absence of effective slip systems ${ }^{1}$ and is manifested by a very large critical thickness for both $\mathrm{AlGaN}$ and GaInN epilayers grown on $\mathrm{GaN}^{2}$ We have shown that relaxation of tensilely stressed $\mathrm{AlGaN}$ (on $\mathrm{GaN}$ ) proceeds through the propagation of incipient cracks, which in turn activates the slip systems for misfit dislocations around the crack tips. ${ }^{3}$ Even though III-nitride devices are known for their relative insensitivity to a high density of dislocations, ${ }^{4}$ cracking due to the tensile mismatch between $\mathrm{AlGaN}$ and the GaN pseudo-substrate tends to severely deteriorate device performance. ${ }^{5}$ Various types of interlayers, generally referred to as a low-temperature (LT) grown layer inserted between two layers grown at regular, high temperatures (HT), have been reported to influence the structural properties of the III-Nitrides. Nakamura $\mathrm{et} \mathrm{al.}{ }^{6}$ reported the use of an InGaN layer before the growth of AlGaN cladding layers (in purple laser diode structures) to avoid cracking. Iwaya et al. ${ }^{7}$ reported the use of low-temperature (LT) deposited AIN and GaN interlayers for the reduction of threading dislocations. Very recently, Amano et al. ${ }^{8}$ demonstrated that the introduction of a LT AlN interlayer on a high-temperature (HT) grown GaN epilayer facilitates subsequent crack-free growth of HT AlGaN. Since the LT interlayers (typically 100 300 $\AA$ thick) are embedded in thick 
$(>1 \mu \mathrm{m})$ heterostructures, it is very difficult to extract information concerning the strainstate in the interlayers. In this work, we investigate the strain relaxation of $\sim 150-\AA ̊ \AA-$ thick LT AIN, GaN, and ternary-alloy interlayers inserted into HT-GaN/AlGaN heterostructures. LT-interlayer strain was quantified using in-situ film-stress measurements in combination with ex-situ characterization of alloy composition. We found that the in-plane lattice parameter of the partially strain-relaxed LT interlayer can be directly tuned to control and compensate growth stress during subsequent growth of $\mathrm{AlGaN}$ heterostructures of various compositions.

Five samples (labeled A-E herein) were grown by metal-organic vapor-phase epitaxy (MOVPE) on (0001) $\mathrm{Al}_{2} \mathrm{O}_{3}$ substrates. The growth sequence for these samples was: (I) nucleation of $200-\AA$-thick GaN at $550{ }^{\circ} \mathrm{C}$, (II) growth of a $1.0-\mu$ m-thick GaN pseudo-substrate at $1050^{\circ} \mathrm{C}$, (III) followed by a $150-\AA$-thick LT-AlGaN interlayer deposited at $700{ }^{\circ} \mathrm{C}$, and (IV) capped with a $0.9-\mu \mathrm{m}$-thick $\mathrm{Al}_{0.2} \mathrm{Ga}_{0.8} \mathrm{~N}$ layer again grown at $1050{ }^{\circ} \mathrm{C}$ (all layers Si-doped, $\mathrm{n} \sim 2 \times 10^{18} \mathrm{~cm}^{-3}$ ). The schematics of layering and growth sequence are shown in Figure 1. The composition of the $\mathrm{LT} \mathrm{Al}_{x} \mathrm{Ga}_{1-x} \mathrm{~N}$ interlayer for samples A-E was intentionally varied from $x_{L T}=0(\mathrm{GaN})$ to $x_{L T}=1(\mathrm{AlN})$, respectively; the samples are otherwise similar. In addition to using the binary AlN interlayers as was reported previously ${ }^{8}$, we extended our investigation into ternary $\mathrm{AlGaN}$ interlayers based on two considerations: 1) AlN is normally insulating and may not be compatible with devices requiring vertical current transport, and 2) the employment of $\mathrm{AlGaN}$ should render direct tunability in the in-plane lattice parameter. Our growth procedures for $\mathrm{GaN}$ on sapphire and a description of the MOVPE reactor appear elsewhere. ${ }^{9}$ The LT AlGaN interlayers and $\mathrm{HT} \mathrm{AlGaN}$ layers of present interest were grown at 40 Torr. $\mathrm{NH}_{3}$ and $\mathrm{H}_{2}$ 
flows were set at 2.5 and $5 \mathrm{l} / \mathrm{min}$, respectively. Trimethylgallium and trimethylaluminum were employed as metal-organic precursors.

The Al composition of the thin LT interlayers $\left(x_{L T}\right)$ was determined by Rutherford backscattering spectrometry (RBS) ${ }^{10}$ using a $2.5 \mathrm{MeV}{ }^{4} \mathrm{He}^{+}$ion beam. The RBS was performed on a separate set of $400-\AA$-thick LT-AlGaN calibration samples grown in' the same manner as samples A-E, but without HT-AlGaN capping layers. The composition of the much thicker HT AlGaN layers $\left(x_{H T}\right)$ of samples A-E was directly measured using high-resolution x-ray diffractometry (XRD). Symmetric (0002) and asymmetric (20-24) reflections were used to determine both in-plane $(a)$ and out-of-plane (c) lattice parameters. Once the strained $a$ and $c$ lattice parameters were measured, corresponding unstrained lattice parameters, elastic strains, and $\mathrm{Al}$ compositions were computed from elasticity theory ${ }^{11}$ under the assumption that Vegard's law holds for the unstrained-lattice parameters $^{12}$ and elastic constants. ${ }^{13}$ The resulting RBS (for $x_{L T}$ ) and XRD (for $x_{H T}$ ) compositions are listed in Table I.

Real time in-situ stress monitoring based on wafer-curvature measurements was performed with a multi-beam optical stress sensor (MOSS) ${ }^{14}$ modified for use on our reactor. To determine the wafer curvature, the divergence of multiple initially parallel laser beams is measured on a CCD camera after reflection of the beams from the film/substrate surface. Changes in wafer curvature induce a proportional change in the beam spacing on the camera. This technique provides a direct measurement of the stressthickness product during MOVPE. ${ }^{15}$ Slopes of the (stress)x(thickness) traces versus time during deposition cycles can be converted to instantaneous stress once the time scale is 
converted to thickness scale using growth rates derived from in-situ reflectance measurements. ${ }^{15}$

The evolution of growth stress was measured by MOSS and the results are shown in Fig. 2. Similar to our previous observations, ${ }^{3}$ the growth of GaN on LT GaN buffers (Region II in Fig. 2) exhibited a characteristic residual tensile stress $\left(\sigma_{G a N}\right)$ that varies between 0.35 and $0.56 \mathrm{GPa}$ (see Table I). A much wider difference, on the other hand, was observed in the variation of the initial growth stress $\left(\sigma_{H T-A l G a N}\right)$ during growth of HT AlGaN layers (Region IV in Figure 2) on the interlayers. Very steep positive slopes, accompanied by successive step-wise stress relaxation, were seen in Samples A and B. As the $\mathrm{Al}$ concentration in the $\mathrm{LT} \mathrm{AlGaN}$ interlayers was increased from $\mathrm{A}(\mathrm{GaN})$ to $\mathrm{E}$ (AIN), the magnitude of the tensile stress of the HT AlGaN gradually decreased. The sample grown using a binary LT AIN interlayer, Sample E, exhibited a negative slope indicative of an initial compressive stress.

Surface-cracking of samples A-E was examined using a differential interferencecontrast Nomarski microscope. We also calculated the critical thickness for cracking $\left(h_{g}\right)$ of the HT AlGaN using the initial stress $\left(\sigma_{H T-A l G a N}\right)$ produced by growth on the partially relaxed LT interlayers on HT GaN. The results for $h_{g}$ given by Griffith's criterion for crack propagation ${ }^{3}$ are compared with the Nomarski micrographs in Figure 3. Recalling that the $\mathrm{HT} \mathrm{Al}_{0.2} \mathrm{Ga}_{0.8} \mathrm{~N}$ is $0.9 \mu \mathrm{m}$ thick, we find consistent results. As expected, the LT GaN interlayer, which produces no relaxation of HT-AlGaN coherency stress, has a critical thickness for fracture much smaller than the HT-layer thickness. The result is the highest degree of cracking as seen in Fig 3(a). Addition of intermediate amounts of $\mathrm{Al}$ $\left(x_{L T}=0.35-0.42\right)$ to the LT interlayer reduces the HT-AlGaN coherency stress and 
increases $h_{g} \sim$ fourfold. Cracking is still observed in Figs. 3(b) and 3(c) since the HTAlGaN thickness is still twice of $h_{g}$. Increasing the LT-interlayer composition to $x_{L T}=0.62$ raises $h_{g}$ by $\sim$ thirtyfold -- now far above the HT-AlGaN thickness. Examining Fig. 3(d), the $\mathrm{HT}-\mathrm{Al}_{0.2} \mathrm{Ga}_{0.8} \mathrm{~N}$ growth is appropriately crack-free. Finally, as the LT interlayer becomes pure $\mathrm{AIN}$, the $\mathrm{HT} \mathrm{AlGaN}$ moves into compression; crack propagation can no longer be driven by composition-induced stress at any layer thickness as seen in Fig. 3(e).

A key parameter in employing the interlayer scheme is the degree of pseudomorphism of the LT interlayers. Information of the in-plane lattice constants of the interlayers immediately before the nucleation of $\mathrm{HT}$ AlGaN layers is requred to determine the degree of coherency. This lattice information is replicated during initial and presumably coherent growth of HT AlGaN layers and can be "captured" with the availability of the in-situ stress sensor before relaxation takes place; the HT AlGaN test layers thus serve as "probes" to yield the lattice parameters of the underlying interlayers. The methodology of extracting the degree of lattice strain relaxation of the interlayers is presented in the following. In brief, the use of XRD and RBS provide the information of the $\mathrm{Al}$ concentrations in the $\mathrm{HT} \mathrm{AlGaN}\left(x_{H T}\right)$ and $\mathrm{LT} \mathrm{AlGaN}\left(x_{L T}\right)$ interlayers, respectively. The slopes measured via MOSS during the initial growth of HT AlGaN layers and during HT GaN yielded instantaneous growth stresses, $\sigma_{H T-A l G a N}$ and $\sigma_{G a N}$, respectively. The measured stresses were converted to strains $\left(\varepsilon_{H T-A l G a N}\right.$ and $\left.\varepsilon_{G a N}\right)$ with information of the biaxial bulk modulus ( $\varepsilon=\sigma / M, M=449.6 \mathrm{GPa}^{15}$ ). Assuming an initial coherent growth of HT AlGaN test layers on the LT interlayers, the in-plane lattice constant of the $\mathrm{LT} \mathrm{AlGaN}$ interlayers can be expressed as $a_{L T-A l G a N}=a_{H T-A l G a N}=\left(1+\varepsilon_{H T-}\right.$ 
$\left.{ }_{A l G a N}\right) a_{0}\left(x_{H T}\right)$, where $a_{0}\left(x_{H T}\right)$ is the in-plane lattice constant of the free-standing AlGaN. With the knowledge of $a_{L T-A l G a N}, a_{0}\left(x_{L T}\right)$, and $a_{G a N}=\left(1+\varepsilon_{G a N}\right) a_{0}(G a N)$, the fraction of the strain relief can be approximated to be $\left[a_{G a N}-a_{L T-A l G a N}\right] /\left[a_{G a N^{-}} a_{0}\left(x_{L T}\right)\right]$. All the parameters are summarized in Table I.

The fact that $a_{H T-A I G a N}$ for Sample A (the sample with a LT GaN interlayer), which was derived from a combination of stress $\left(\sigma_{H T-A l G a N}\right)$ during $\mathrm{AlGaN}$ growth and $\mathrm{Al}$ concentration $\left(x_{H T}\right)$ from XRD, approaches $a_{G a N}$ lends strong support to both the assumption of an initial coherent HT AlGaN growth and the validity of current treatment combining in-situ and ex-situ characterizations. It is clear from Table I (columns $a_{L T}$. ${ }_{A l G a N}$ and $\left.a_{G a N}\right)$ that crack suppression through stress engineering is based on the ability to vary the in-plane lattice parameters of the LT interlayers. Under the growth conditions employed in this work, the deposition of $\mathrm{LT} \mathrm{Al}_{x} \mathrm{Ga}_{1-\mathrm{x}} \mathrm{N}$ interlayers produces templates with in-plane lattice constants equal to those of free-standing $\mathrm{Al}_{y} \mathrm{Ga}_{1-y} \mathrm{~N}$ with $y \sim x / 3$. The observed partial lattice relaxation in the LT interlayers was possibly accomplished through the nucleation and gliding of edge-dislocations on the basal (0001) plane ${ }^{3}$ between LT interlayers and HT-GaN. The geometrical constraints for nucleation and gliding of misfit dislocations during planar, coherent heteroepitaxy in wurtzite systems ${ }^{1}$ are likely lifted due to the alteration of microstructural properties. Atomic force microscopy performed on as-grown interlayers revealed the presence of non-planar discrete and/or interconnected islands. Such a change in microstructure causes a highly inhomogeneous, non-biaxial distribution of the strain field, which makes the admission of misfit dislocations energetically favorable. Alternatively, strain relaxation of the LT interlayers might occur through initial cracking and subsequent dislocating; the final, 
crack-free HT AlGaN layers (Samples A and B) would then be the result of lateral growth over the cracks. We note that in the cases of crack-free growth on the AlGaN interlayers ( $\mathrm{Al}>60 \%$, Samples $\mathrm{A}$ and $\mathrm{B}$ ), the structural quality of the $0.9 \mu \mathrm{m}$ AlGaN layers was as good as the underlying GaN pseudo-substrates as indicated by the rocking curve linewidths using (0004) diffraction (around $320 \operatorname{arcsec}$ for both $\mathrm{AlGaN}$ and $\mathrm{GaN}$ ).

In conclusion, we found that the use of LT AlGaN interlayers is effective in "redefining" the in-plane lattice parameter through strain relaxation. The interlayers serve to mediate the elastic tensile mismatch between the adjacent layers and extend the cracking critical thickness. It is anticipated that the interlayer scheme will greatly increase the flexibility in the heteroepitaxy of AlGaN-based devices.

The authors acknowledge technical assistance from T. M. Kerley. One of us (KEW) acknowledges support from the General Electric Minority Engineering Faculty Development Initiative fellowship program. Sandia is a multiprogram laboratory operated by Sandia Corporation, a Lockheed Martin Company, for the United States Department of Energy under Contract DE-AC04-94AL85000. 
Figure Captions

Figure 1. (a) Schematic drawing of the layer structure employed in this work, and (b) schematic drawing of the temperature profile during a typical growth of the LT GaN buffer, HT GaN, LT AlGaN interlayer, and HT AlGaN test layers.

Figure 2. (stress)x(thickness) versus time during growth of Samples A-E. Dotted lines serve to demarcate the beginning and end of the HT GaN and HT AlGaN growths. (Automatic power control based on the intensity of reflected beams as feedback was employed during this work. One should focus on the general slopes during HT GaN and HT AlGaN growth and ignore the finer oscillations, which are artifacts possibly due to the power dependence of the laser beam profile.)

Figure 3. Nomarski surface micrograph ( 200X) of Samples A-E. a) $x_{L T}=0.00(\mathrm{GaN})$; b) $x_{L T}=0.34$; c) $x_{L T}=0.42 ;$ d) $x_{L T}=0.62 ;$ e) $x_{L T}=1.00(\mathrm{AIN})$. 


\section{Table Caption}

Table I. Summary of measured and calculated parameters for Samples A-E. (Fraction of strain relief in Sample A is not applicable since a LT GaN interlayer was grown on $\mathrm{GaN}$.) 


\section{References}

${ }^{1}$ X. J. Ning, F. R. Chien, P. Pirouz, J. W. Yang, and M. Asif Khan, J. Mat. Res. 11, 580 (1996).

${ }^{2}$ I. Akasaki and H. Amano, Jpn. J. Appl. Phys. 36, 5393 (1997).

${ }^{3}$ S. J. Hearne, J. Han, S. R. Lee, J. A. Floro, D. M. Follstaedt, E. Chason, and I. S. T. Tsong, Appl. Phys. Lett. 76, 1534 (2000).

${ }^{4}$ S. D. Lester, F. A. Ponce, M. G. Craford, D. A. Steigerwald, Appl. Phys. Lett. 66, 1249 (1995).

${ }^{5}$ J. Han, M. H. Crawford, R. J. Shul, S. J. Hearne, E. Chason, J. J. Figiel, and M. Banas, MRS Internet J. Nitride Semicond. Res. 4S1, G7.7 (1999).

${ }^{6}$ S. Nakamura and G. Fasol, The Blue Laser Diode, p.277, Springer-Verlag, Berlin (1997)

${ }^{7}$ M. Iwaya, T. Takeuchi, S. Yamaguchi, C. Wetzel, H. Amano, and I. Akasaki, Jpn. J. Appl. Phys. 37, L316 (1998).

${ }^{8}$ H. Amano, M. Iwaya, N. Hayashi, T. Kashima, S. Nitta, C. Wetzel, and I. Akasaki, phys. stat. Sol. (b) 216,683 (1999).

${ }^{9}$ J. Han, T. -B. Ng, R. M. Biefeld, M. H. Crawford, and D. M. Follstaedt: Appl. Phys. Lett. 71 (1997) 3114.

${ }^{10}$ W-K. Chu, J. W. Mayer, and M.-A. Nicolet: Backscattering Spectrometry (Academic, New York, 1978).

${ }^{11} \Delta c / c=-2\left(C_{13} / C_{33}\right) \Delta a / a$ for a wurtzite crystal under biaxial strain along (0001).

12 A. Trampert, O. Brandt, and K. H. Ploog, Galium Nitride (GaN) I, p174 175, Academic Press, San Diego (1998).

${ }^{13}$ A. F. Wright, J. Appl. Phys. 82, 482 (1997).

${ }^{14}$ C. Taylor, D. Barlett, E. Chason, J. A. Floro, Ind. Physicist 4, 25 (1998)

${ }^{15}$ S. Hearne, E. Chason, J. Han, J. A. Floro, J. Hunter, J. J. Figiel: Appl. Phys. Lett. 74 (1999) 356 


\begin{tabular}{|c|}
\hline $\begin{array}{c}\text { IV. HT AIGaN test } \\
\text { layer }(0.9 \mu \mathrm{m})\end{array}$ \\
\hline III. LT AIGaN interlayer \\
\hline II. HT GaN $(1 \mu \mathrm{m})$ \\
\hline I. LT GaN buffer $(200 \mathrm{~A})$ \\
\hline \\
Sapphire \\
\hline
\end{tabular}

(a)

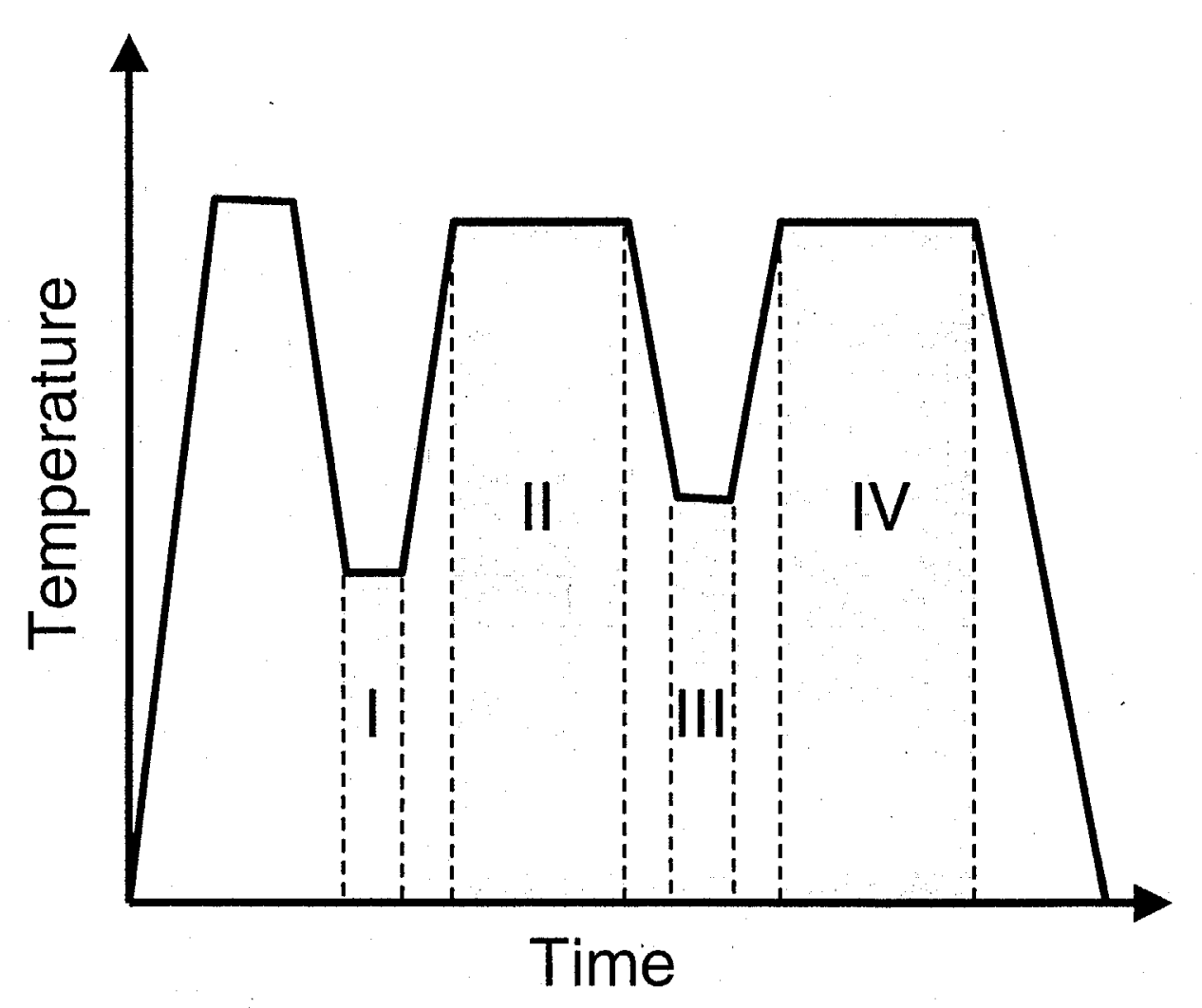

(b)

Fig 1. Han et al. 


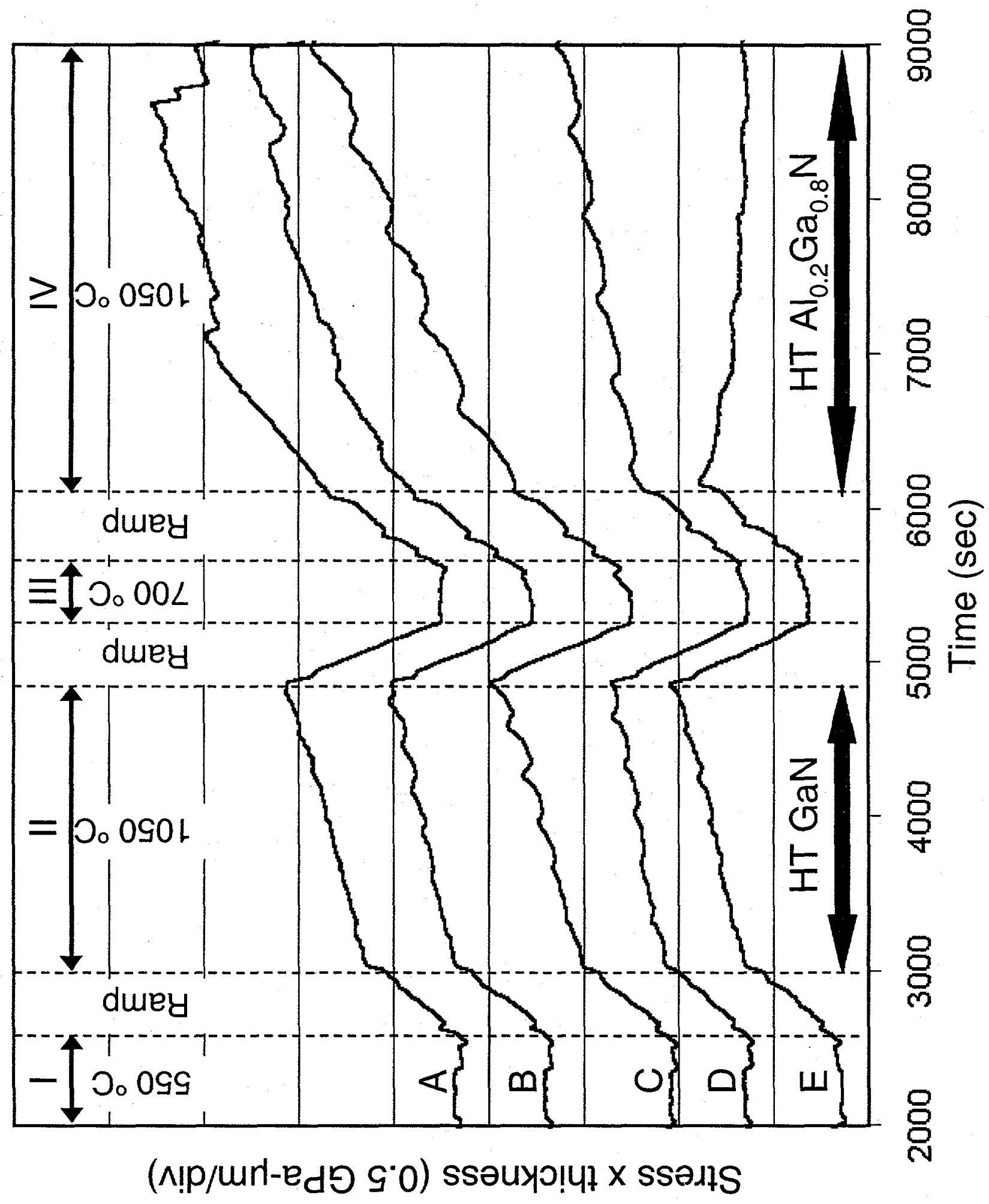




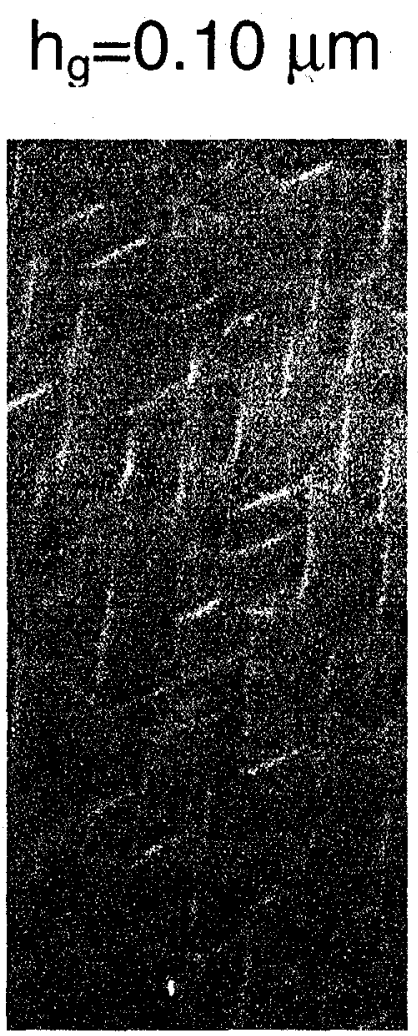

(a) $x_{L T}=0$

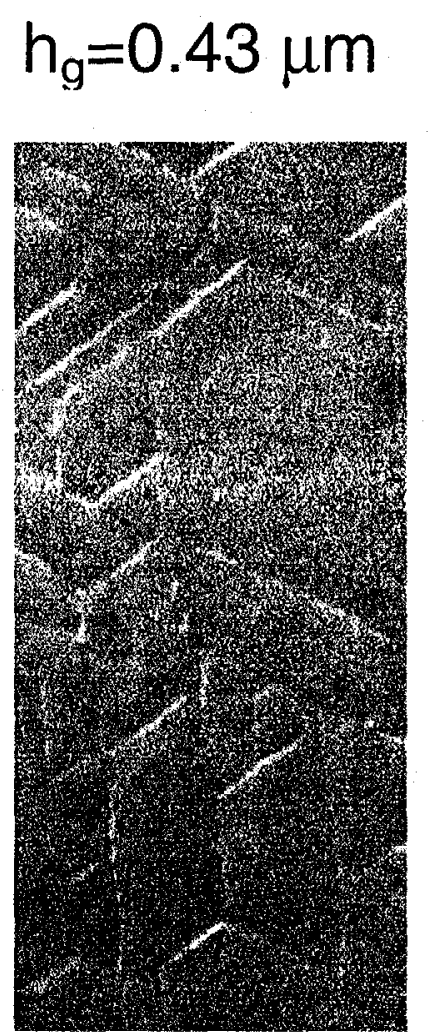

(b) $x_{L T}=0.34$ $h_{g}=0.38 \mu \mathrm{m}$

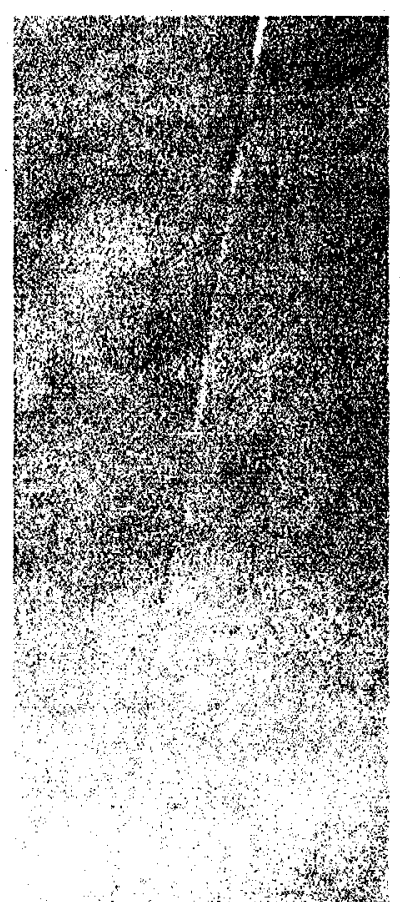

(c) $x_{L T}=0.42$ $h_{g}=3.0 \mu \mathrm{m}$

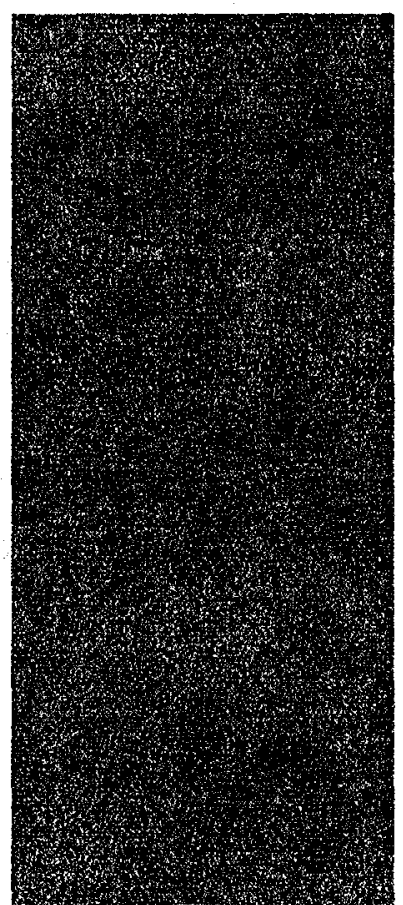

(d) $x_{L T}=0.62$ $h_{g}=\infty$

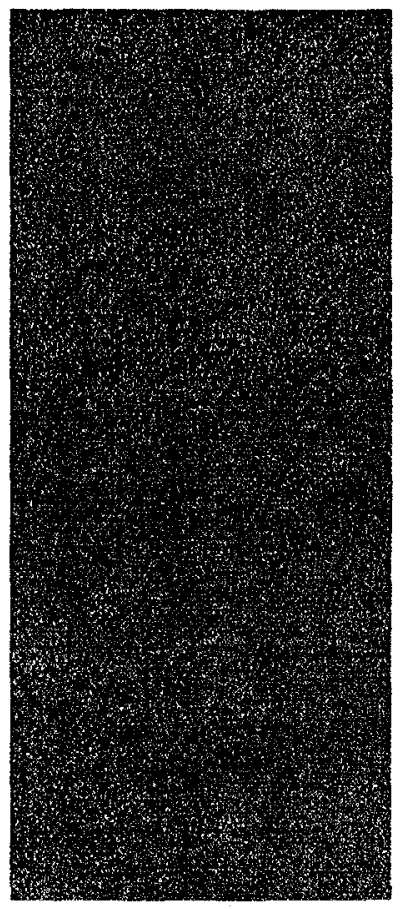

(e) $x_{L T}=1$

Fig 3. Han et al. 


\begin{tabular}{ccccccccccc} 
Sample & $x_{H T}$ & $\begin{array}{c}\sigma_{H T \cdot A l G a N} \\
(\mathrm{GPa})\end{array}$ & $\begin{array}{c}\varepsilon_{H T-A l G a N} \\
(\%)\end{array}$ & $\begin{array}{c}a_{H T-A l G a N} \\
a_{L T \text {-AlGaN }}(\AA)\end{array}$ & $\begin{array}{c}\sigma_{G a N} \\
(\mathrm{GPa})\end{array}$ & $\begin{array}{c}\varepsilon_{G a N} \\
(\%)\end{array}$ & $\begin{array}{c}a_{G a N} \\
(\AA)\end{array}$ & $\begin{array}{c}x_{L T} \\
a_{0}\left(x_{L T}\right) \\
(\AA)\end{array}$ & $\begin{array}{c}\text { Fraction of } \\
\text { strain relief }\end{array}$ \\
\hline \hline A & 0.201 & 2.730 & 0.607 & 3.1930 & 0.448 & 0.100 & 3.1922 & 0.00 & 3.1890 & NA \\
B & 0.203 & 1.310 & 0.291 & 3.1828 & 0.393 & 0.087 & 3.1918 & 0.34 & 3.1631 & 0.314 \\
C & 0.201 & 1.400 & 0.311 & 3.1836 & 0.567 & 0.126 & 3.1930 & 0.42 & 3.1570 & 0.262 \\
D & 0.200 & 0.501 & 0.111 & 3.1773 & 0.355 & 0.079 & 3.1915 & 0.62 & 3.1418 & 0.286 \\
E & 0.200 & -0.746 & -0.166 & 3.1685 & 0.459 & 0.102 & 3.1923 & 1.00 & 3.1129 & 0.299
\end{tabular}

